\title{
Fabrication of Biodegradable Disc-shaped Microparticles with Micropattern using a Hot Embossing Process with Porous Microparticles
}

\author{
Jiyea Hwang, Young Bin Choy ${ }^{1}$, Soonmin Seo and Jung-Hwan Park ${ }^{\dagger}$ \\ College of BioNano Technology and Gachon BioNano Research Institute, Kyungwon University, \\ Seongnam, Geonggi-do, 461-701, Korea \\ ${ }^{1}$ Department of Biomedical Engineering, College of Medicine and Institute of Medical \& Biological Engineering, \\ Medical Research Center, Seoul National University, Seoul, 110-799, Korea \\ (Received March 25, $2011 \cdot$ Revised April 18, $2011 \cdot$ Accepted April 24, 2011)
}

\begin{abstract}
This paper demonstrates the development of a method for preparing micropatterned microdiscs in order to increase contact area with cells and to change the release pattern of drugs. The microdiscs were manufactured with hot embossing, where a polyurethane master structure was pressed onto both solid and porous microparticles made of polylacticco-glycolic acid at various temperatures to form a micropattern on the microdiscs. Flat microdiscs were formed by hot embossing of porous microparticles; the porosity allowed space for flattening of the microdiscs. Three types of microgrooves were patterned onto the flat microdiscs using prepared micropatterned molds: (1) $10 \mu \mathrm{m}$ deep, $5 \mu \mathrm{m}$ wide, and spaced $2 \mu \mathrm{m}$ apart; (2) $10 \mu \mathrm{m}$ deep, $9 \mu \mathrm{m}$ wide, and spaced $5 \mu \mathrm{m}$ apart; and (3) $10 \mu \mathrm{m}$ deep, $50 \mu \mathrm{m}$ wide, and spaced $50 \mu \mathrm{m}$ apart. This novel microdisc preparation method using hot embossing to create micropatterns on flattened porous microparticles provides the opportunity for low-cost, rapid manufacture of microdiscs that can be used to control cell adhesion and drug delivery rates.
\end{abstract}

Key words - Biodegradable polymer, Porous microparticle, Embossing, Micropatterning, Microdisc

Biodegradable microparticles made of poly-lactic-co-glycolic acid (PLGA) have been used for sustained parenteral, pulmonary, and ocular delivery of drugs and as carriers for immunization applications (Jiang et al., 2005; Sinha and Trehan, 2003). PLGA degrades by hydrolysis of its ester linkages in the presence of water and is approved as a therapeutic device by the FDA because of its biocompatibility and biodegradability (Luo et al., 1999). Biodegradable polymer particles (e.g., microspheres, microcapsules, and nanoparticles) are highly useful because they can be administered to a variety of locations in vivo through a syringe needle. There are several methods for preparation of biodegradable microparticles, such as emulsion, phase separation, spray drying, and in situ polymerization (Vehring, 2008). However, conventional fabrication methods have limitations in controlling the shape and geometry of the microparticles. Usually biodegradable microparticles have a spherical shape, and the release pattern of a drug from microparticles has been controlled by composition of the polymer, content of the drug, and rate-controlling additives (Jeong et al., 2000).

Soft lithography techniques provide a method to avoid the

'Corresponding Author:

Tel : +82-31-750-8551, E-mail : pa90201@kyungwon.ac.kr

DOI : $10.4333 /$ KPS.2011.41.3.147 limitations of conventional microfabrication and complement it to fabricate polymeric microdevices for drug delivery (Shawgo et al., 2002). Soft lithography is a general term for a micro/ nonphotolithographic method based on replica molding using poly-di-methyl-siloxane (PDMS), silicon, and thermoplastics for micro- and nanofabrication. The use of the micromolding process can transfer patterns with high resolution ranging from a few nanometers to a few hundred microns (Mi et al., 2006). Hot embossing, a nanoimprint lithography technique, has been gaining attention as an alternative candidate for next-generation patterning technologies because it has the advantages of simplicity and low cost compared to conventional photolithographic techniques. The hot embossing process is advantageous for producing particles with various geometries. These flat particles have a relatively large surface area for cell or tissue binding and a different release pattern compared with spherical microparticles (Charest st al., 2004, Choy et al., 2008).

The purpose of this study was to develop a novel fabrication method using micro/nano patterning technology to fabricate flattened, disc-shaped microparticles with micro/nano patterns as controlled drug delivery devices. We used a micromolding process to make discs with micropatterns as a means to control tissue response and the drug release rate (Choy et al., 2008). For mass production, patterned microdiscs should be fabricated by an easy process. The conditions for preparing micro- 
patterned discs and the parameters that determined the geometry of microdiscs were studied.

\section{Materials and Methods}

\section{Preparation of solid and porous PLGA microparticles}

PLGA microparticles were prepared using the emulsion method. An initial solution containing methylene chloride and PLGA polymer (LACTEL, DURECT Corp. Pelham, AL) was prepared at room temperature. The concentration of the PLGA solution was $5 \%(\mathrm{w} / \mathrm{w})$. The polymer solution was emulsified in water using mechanical stirring at room temperature for 5 min at $500 \mathrm{rpm}$ (HA 1000-3, Hanil, Korea). Deionized water $(100 \mathrm{~mL})$ with $0.1 \%$ Tween $80(\mathrm{w} / \mathrm{w})$ was added to the resulting mixture and magnetically stirred for an additional $10 \mathrm{~min}$. Finally, the microparticles were passed through a $0.45-\mu \mathrm{m}$ filter (HVLP type, Millipore SA, Saint Quentin Yvelines, France), washed with deionized water five times, and freeze-dried (RP2V Serail SGD, Argenteuil, France). The dried particles were stored at $4^{\circ} \mathrm{C}$. The microparticles were then filtered through a sieve to form particles ranging from $50 \mu \mathrm{m}$ to $200 \mu \mathrm{m}$ in diameter.

Salt particles (Sodium Chloride, Sigma-Aldrich, St. Louis, MO) were pulverized and then passed through a filter (Nylon Net filter, Millipore) to make them less than $20 \mu \mathrm{m}$ in diameter. The salt particles were mixed with the PLGA solution $(1: 4=$ salt : PLGA). Then the salt particles were leached out of the PLGA solution by selective dissolution in deionized water for $12 \mathrm{~h}$ at room temperature.

\section{Preparation of micropattern}

A silicon wafer $300-350 \mu \mathrm{m}$ thick (Nova Electronic Inc., Richadson, TX) was cleaned in 5 parts deionized water, 1 part $30 \%$ hydrogen peroxide (J.T. Baker, Phillipsburg, NJ) and 1 part $27 \%$ ammonium hydroxide (J.T. Baker) at $80^{\circ} \mathrm{C}$ for $15 \mathrm{~min}$ to remove organic residue and then the wafer was dried in a drying oven (Blue M Electric, Watertown, WI) at $120^{\circ} \mathrm{C}$ for 20 min. SU-8 epoxy photoresist with photoinitiator (SU-8100, MicroChem, Newton, MA) was coated onto a wafer The sample was then soft-baked for $10 \mathrm{~h}$ at $100^{\circ} \mathrm{C}$ to remove all the solvent from the epoxy. Then UV light (365 nm, 7000 mJ) was exposed through the circular transparent regions of an optical mask with 5,9 , and $50 \mu \mathrm{m}$ of line width and the same dimensions of center-to-center spacing as lines onto the SU-8 with a photo initiator using a mask aligner (OAI: Optical Associates, Inc., San Jose, CA). The UV-exposed part became crosslinked. Next, the sample was post-baked to cross-link the SU8 on a hotplate for $30 \mathrm{~min}$ at $100^{\circ} \mathrm{C}$. After cooling, the noncross-linked SU-8 was then developed with propyl glycol methyl ether acetate (PGMEA, Sigma-Aldrich). A UV curable poly(urethaneacrylate) (PUA, MINS101m, Minuta Tech.) structure was replicated from the SU- 8 structures. The resulting film had a height of 5, 9, and $50 \mu \mathrm{m}$ line and space 2, 5 and $50 \mu \mathrm{m}$ space patterns respectively.

\section{Preparation of microdisc}

Prepared microparticles were placed on the glass slides and spread evenly. Then a sample slide was covered with slide glass. Samples were put on the mounting press of a heating press (MBM, Gimpo, Korea) and left until they were heated to the determined temperature. The PLGA solid and porous microparticles were pressed in the hot compression mounting at $2,4,6$, and 8 bar at $60^{\circ} \mathrm{C}$ for $5 \mathrm{sec}$ to investigate the flattening from sphere to disk as a result of the pressure. Porous PLGA microparticles were pressed at various temperatures of $25,30,45$, and $60^{\circ} \mathrm{C}$ at 6 bar for $5 \mathrm{sec}$ to observe the flattening resulting from the temperature. a
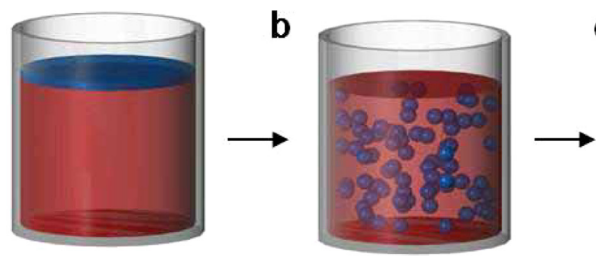

C
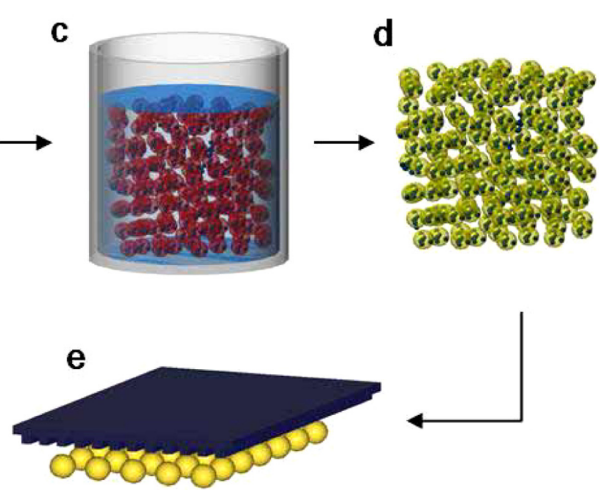

Figure 1. Fabrication process of microdiscs with micropatterns from porous microparticles. (a) a immisible solution containing PLGA solution and water, (b) the polymer solution emulsified in water, (c) organic solvent removal, (d) solidified PLGA microparticles, (e) hot embossing of PLGA microparticles, (f) microdisc 

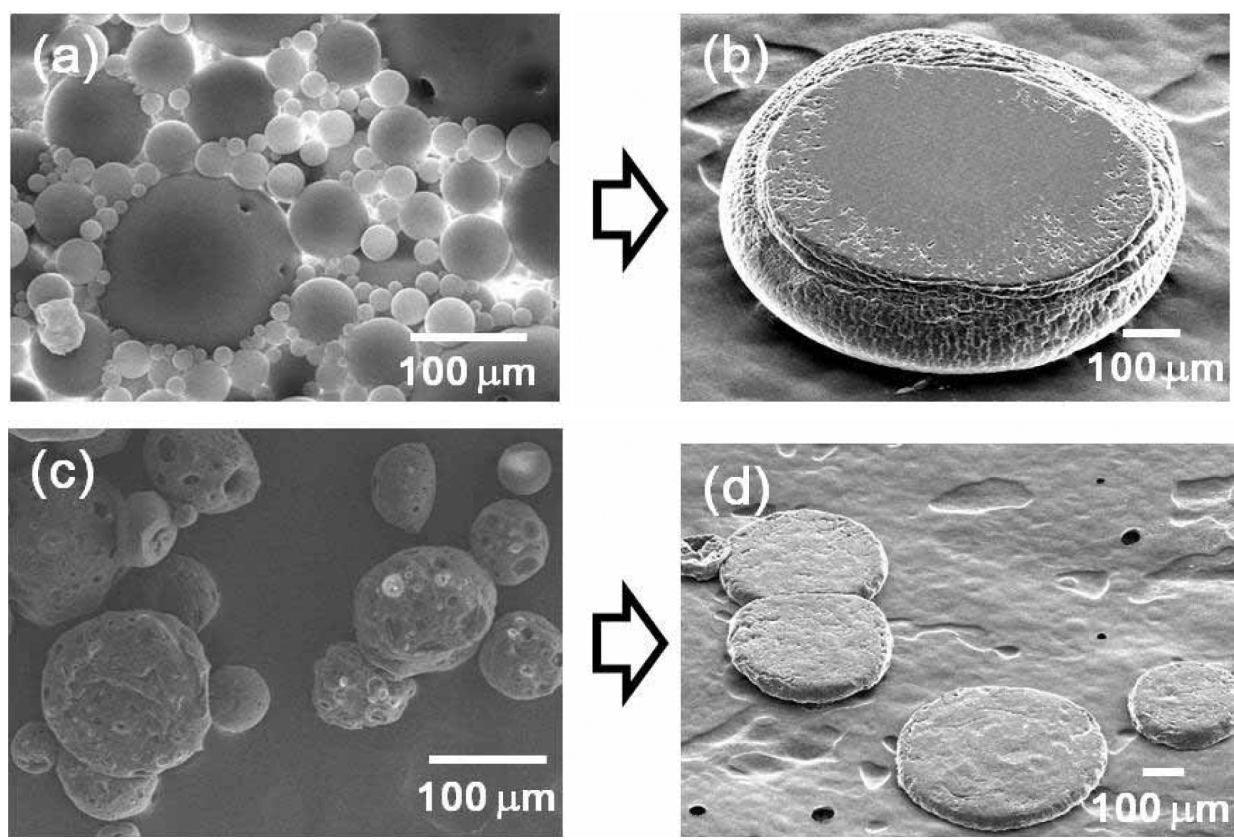

Figure 2. Scanning electron microscopy images of (a) solid PLGA microparticles, (b) microdisc from solid microparticles by hot embossing, (c) porous PLGA microparticles and (d) microdisc from porous microparticles by hot embossing.

\section{Particle characterization}

Microparticles were observed by optical microscopy (BH2, Olympus, Tokyo, Japan). The surface and the internal morphology of the microparticles were investigated by using scanning electron microscopy (SEM; JSM 6310F, JEOL, Paris, France). Freeze-dried samples were mounted onto metal stubs using double-sided adhesive tape, vacuum-coated with a film of carbon (10-nm thick) using a MED 020 (Bal-Tec, Balzers, Lichtenstein).

\section{Results and Discussion}

As shown in Figure 2, spherical solid PLGA microparticles were prepared using an emulsion method. The diameter of the initial particles ranged between $10 \mu \mathrm{m}$ and $200 \mu \mathrm{m}$ before filtering. Porous PLGA microparticles were also prepared using the same process as that for solid microparticles. The content of salt in PLGA particles is $20 \%$ (w/w) and their void fraction may be important to determine the degree of flattening. Two types of microparticles had a diameter ranging from $50 \mu \mathrm{m}$ to $200 \mu \mathrm{m}$ after filtering. After hot embossing of microparticles with a high-pressure press device, the thickness of the microdiscs differed between those formed from solid microparticles and those formed from porous microparticles. As mentioned above, the goal of this study was to fabricate microdiscs in order to increase contact area with cells and to control drug release rate by a simple and economic process. Empty space in

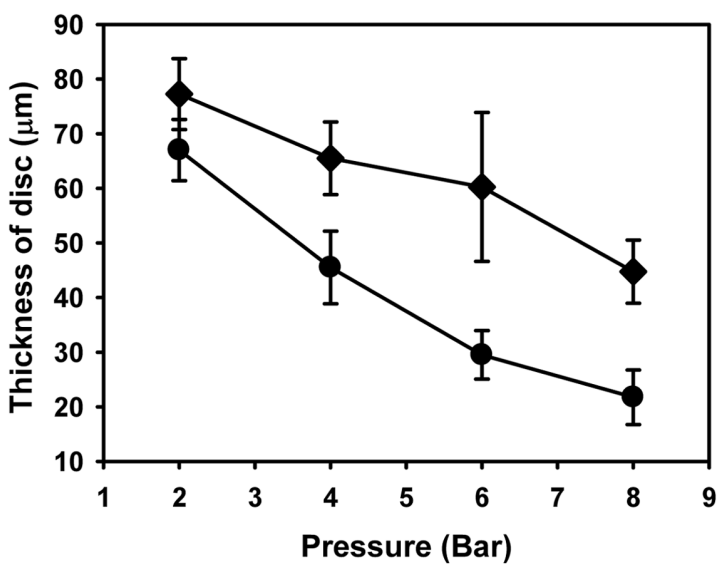

Figure 3. Comparison of thickness change of microdiscs from solid microparticles $(\bullet)$ and porous microparticles $(\boldsymbol{\bullet})$ as a result of embossing pressure.

porous microparticles provides more room to form thinner microdiscs. As shown in Figure 2, the final thickness of microdiscs formed from spherical solid microparticles and porous microparticles with diameters ranging from 50 to $150 \mu \mathrm{m}$ was approximately 60 and $25 \mu \mathrm{m}$, respectively, after compressing particles under 6 bar at $60^{\circ} \mathrm{C}$ for $5 \mathrm{sec}$. The thickness varied depending on the size of particles, which have a bumper function. If the size of the microparticles were uniform, the difference in flattening of the microparticles would be shown clearly.

The change in thickness of microparticles ranging from 

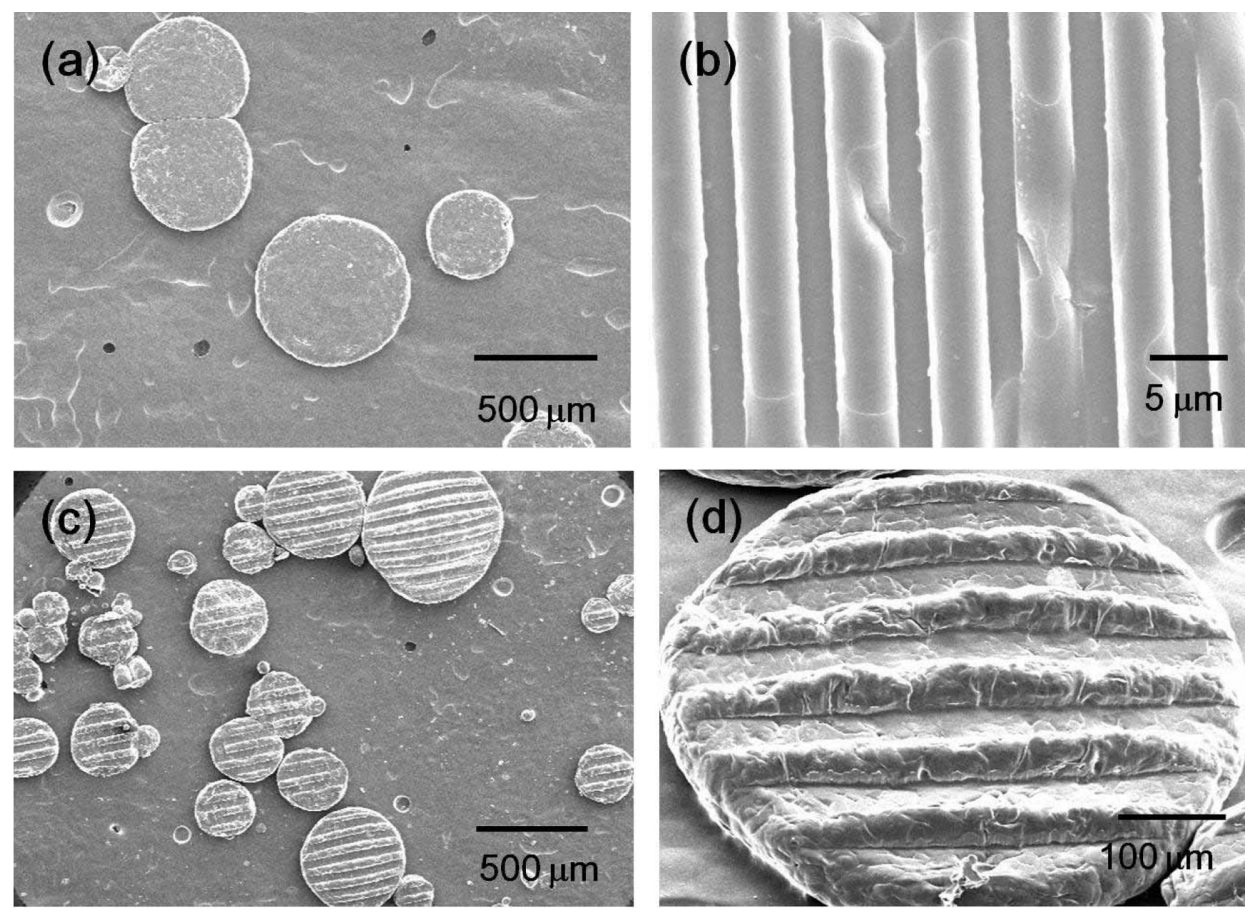

Figure 4. Scanning electron microscopy images of microdiscs (a), (b) with $5 \mu \mathrm{m}$ of line width from low magnification to high magnification and (c), (d) with $50 \mu \mathrm{m}$ of line width from low magnification to high magnification.

$80 \mu \mathrm{m}$ to $150 \mu \mathrm{m}$ in diameter was measured and averaged after hot embossing. The thickness of microdiscs decreased with increased compression pressure, but greater deformation of porous microparticles was performed, as shown in Figure 3. These data showed that the preparation of microdiscs from porous microparticles using hot embossing was effective in controlling the geometry of the microdiscs, and porous microparticles can provide much thinner microdiscs than solid microparticles, resulting in different movement and drug delivery properties.

A micropattern was successfully transferred from the PUA mold onto the PLGA microdiscs, as shown in Figure 4. Grooves with $5 \mu \mathrm{m}$ of width are not shown in low magnification because of their small size, but the patterned grooves are shown by high magnification of SEM (Figure 4(b)). However, grooves with $100 \mathrm{~nm}$ of width (Figure 5) were rarely replicated due to the high resolution under the parameters of this study (data not shown). To transfer the nanoscaled pattern onto the microdiscs, an optimal process should be performed, including increasing the process time and the embossing pressure. The patterning process has been used for fabricating various kinds of films to control surface adhesion and optical properties. Here we utilized the micro/nano hot embossing of porous microparticles to control the drug delivery property and membrane interaction of biodegradable polymeric microdiscs. This method can provide easy fabrication of microdiscs with

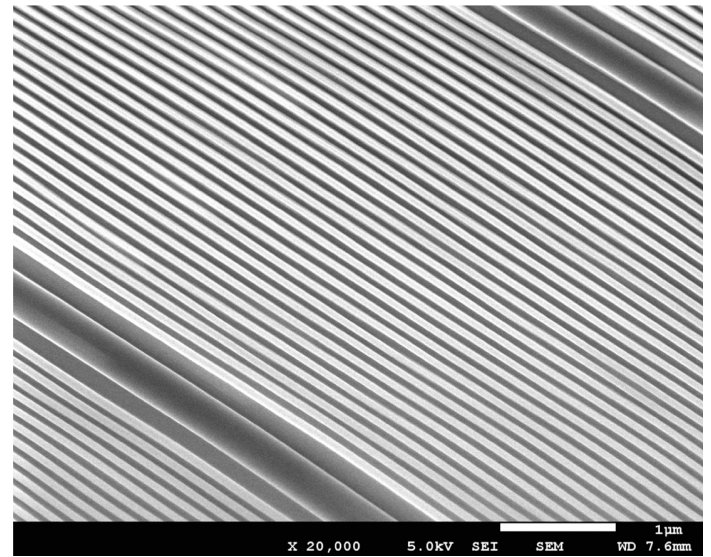

Figure 5. Scanning electron microscopic images of submicron pattern of PUA prepared by using microfabrication process.

various surface and drug delivery properties.

The ratio of patterned area to cross-sectional area was dependent on process temperature. The glass transition temperature of PLGA is between 40 and $45^{\circ} \mathrm{C}$, and the process temperature is important to make microparticles soft enough for flattening. Below the glass transition temperature, as shown in Figure 6(b), only a partial area was patterned and the flattening of microdiscs was insignificant. Above the glass transition temperature, most of the microdisc area was patterned, but the pattern was not transferred at the edge of the microdisc (Figure 6(c)). As shown in Figure 6(d), the entire pattern was 

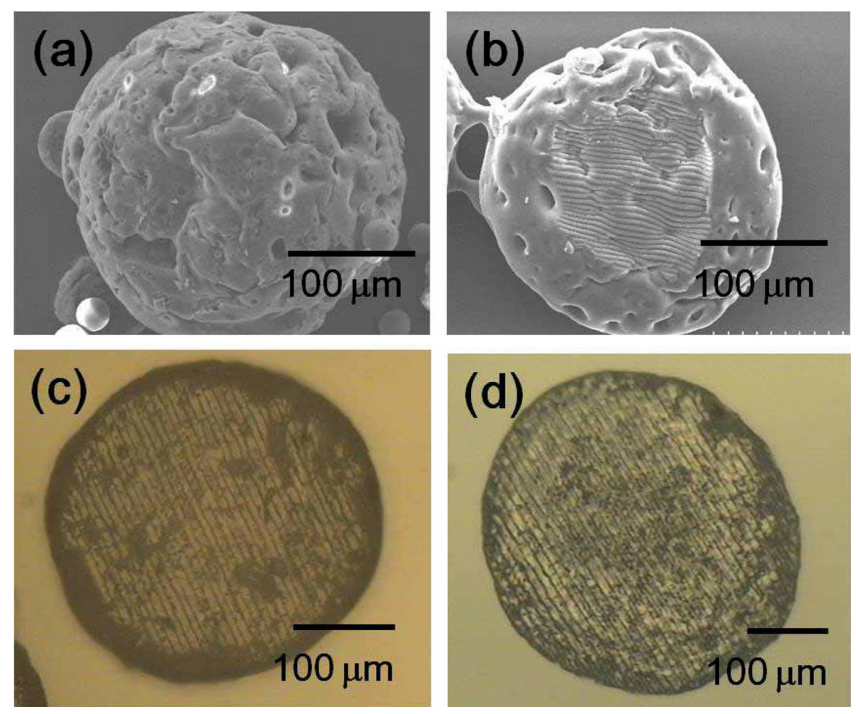

Figure 6. Scanning electron microscopy and optical microscopic images of microdiscs. (a) Porous microparticles, (b) microdisc by hot embossing of porous microparticles at $30^{\circ} \mathrm{C}$, (c) at $45^{\circ} \mathrm{C}$ and (d) at $60^{\circ} \mathrm{C}$ under 6 bar of compression for 5 sec.

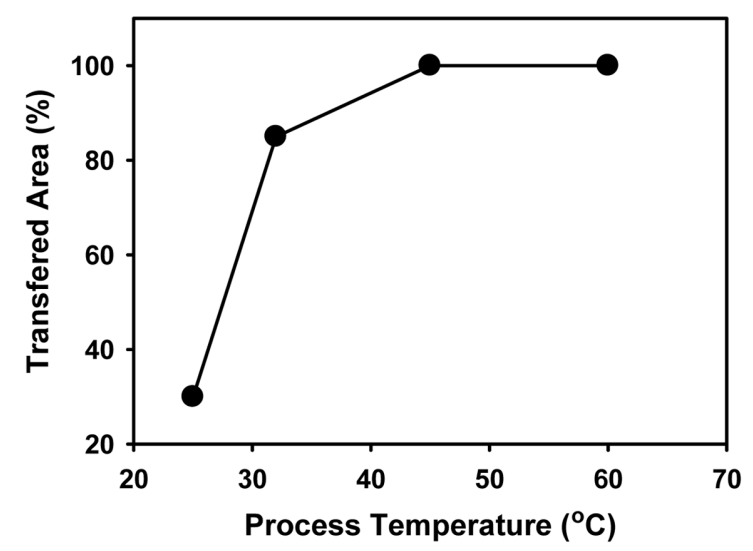

Figure 7. The ratio of patterned area to cross-sectional area as a function of process temperature under 6 bar of embossing pressure.

transferred at $60^{\circ} \mathrm{C}$ under 6 bar of compression pressure for $5 \mathrm{sec}$, showing the temperature increase required for total flattening and patterning of microparticles. If thermally sensitive material is encapsulated in microparticles, embossing at a lower temperature under higher pressure for a short time may be recommended. As summarized in Figure 7, the patterned area increased with the increase in temperature. As expected, the glass transition temperature of polymer was critical to determine the successful formation of grooves on microdiscs.

\section{Acknowledgements}

This work was supported by the GRRC program of Gyeonggi province [GRRC Kyungwon 2010-A01, Development of Microfluidic Chip for diagnosis of disease].

\section{References}

Charest, J.L., Bryant, L.E., Garcia, A.J., King, W.P., 2004. Hot embossing for micropatterned cell substrates. Biomaterials 25, 4767-4775.

Choy, Y.B., Park, J.H., McCarey, B.E., Edelhauser, H.F., Prausnitz, M.R., 2008. Mucoadhesive microdiscs engineered for ophthalmic drug delivery: effect of particle geometry and formulation on preocular residence time. Invest. Ophthalmol. Vis. Sci. 49, 4808.

Jeong, B., Bae, Y.H., Kim, S.W., 2000. Drug release from biodegradable injectable thermosensitive hydrogel of PEGPLGA-PEG triblock copolymers. J. Control Rel. 63, 155-163.

Jiang, W., Gupta, R.K., Deshpande, M.C., Schwendeman, S.P., 2005. Biodegradable poly (lactic-co-glycolic acid) microparticles for injectable delivery of vaccine antigens. Adv. Drug Del. Rev. 57, 391-410.

Luo, D., Woodrow-Mumford, K., Belcheva, N., Saltzman, W.M., 1999. Controlled DNA delivery systems. Pharm. Res. 16, 1300-1308.

Mi, Y., Chan, Y., Trau, D., Huang, P., Chen, E., 2006. Micromolding of PDMS scaffolds and microwells for tissue culture and cell patterning: A new method of microfabrication by the self-assembled micropatterns of diblock copolymer micelles. Polymer 47, 5124-5130.

Shawgo, R.S., Richards Grayson, A.C., Li, Y., Cima, M.J., 2002. BioMEMS for drug delivery. Curr. Opin. Solid State Mater. Sci. 6, 329-334.

Sinha, VR, Trehan, A., 2003. Biodegradable microspheres for protein delivery. J. Control Rel. 90, 261-280.

Vehring, R., 2008. Pharmaceutical particle engineering via spray drying. Pharm. Res. 25, 999-1022. 